\title{
FRACTIONAL STATISTICS \\ IN LOW-DIMENSIONAL SYSTEMS - RANDOM-PHASE APPROXIMATION FOR ANYONS AT FINITE TEMPERATURES
}

\author{
L. JACAK AND P. SitKo \\ Institute of Physics, Technical University of Wroclaw \\ Wyb. Wyspiańskiego 27, 50-370 Wroclaw, Poland \\ (Received December 14, 1992; in final form March 1, 1993)
}

\begin{abstract}
The non-zero temperature theory for non-interacting anyon gas is developed within the random-phase approximation. It is proved that the phase transition superconducting normal state of anyons does not occur and the Meissner effect disappears at non-zero temperatures. The mutual correspondence of new Haldane theory of anyons and mean field treatment is found. A short overview of the fractional statistics theory is also given.
\end{abstract}

PACS numbers: 05.30.-d, 74.20.Kk

\section{Introduction}

Recently new physics of two dimensional systems has emerged $([1,2])$ which may be the key to understanding such phenomena like the fractional quantum Hall effect $([3,4])$ or perhaps also high- $T_{\mathrm{c}}$ superconductivity $([5,6])$. Topological properties turn out to be crucial for considerations of $2 \mathrm{D}$ systems and lead to quite new and exotic phenomena in condensed matter, which are more familiar in the field theory, e.g. the string theory. The simplest example of these field theories is that described by the Lagrangian with the Chern-Simons (Ch.-S.) term ([7, 8])

$$
\Delta L_{\mathrm{Ch}-\mathrm{S}}=\mu \epsilon^{i j k} A_{i} \partial_{j} A_{k},
$$

where $A$ is a gauge field.

It was shown that $\mu=1 / 4 \pi k$ describes a spontaneously fixed flux attached to a charged particle, where $k=1,3,5 \ldots$ correspond to the Hall fluid $([3,4])$ while $k=2,4,6 \ldots$ describe the so-called chiral spin liquid $([4,9,10])$. This model provides unified picture of the fractional Hall effect and a chiral spin liquid two distinct physical realizations of fractional statistics. The existence of the Hall liquid has been experimentally verified while the chiral spin liquid (similarly to another one - an anyon superconductor) is still hypothetical. 
Since in the Ch.-S. term we have only one derivative in 2D case, this term dominates the Maxwell term $f_{\mu \nu} f_{\lambda \sigma} g^{\mu \lambda} g^{\nu \sigma}$ at long distances (note that in 3D the analogous Ch.-S. term - $\epsilon \partial A \partial A$ has the same scale dimensions as the Maxwell term). Moreover, the Ch.-S. term is topological since it involves antisymmetric matrix $\epsilon^{i j k}$ and not the metric tensor $g^{i j}$ (in contrast to the Maxwell term). IIence, those properties of microscopic theory governed by the metric (like disorder or impurities) cannot affect the Ch.-S. term - in other words the topological index is robust against small perturbations.

The presence of the Ch.-S. term implies that the parity $P$ and time reversion $T$ are violated in a spontaneous manner as in anyon superconductors $([5,11])$ or owing to the presence of the external magnetic field as in the Hall fluid ([3]).

\section{Quantum Hall fluid}

We begin with a simple problem of a $2 \mathrm{D}$ spinless electron in an external magnetic field. Without the Pauli term, the Hamiltonian reduces to the oscillator IIamiltonian:

$$
\begin{aligned}
& H=\frac{1}{2 m}\left(-\mathrm{i} \hbar \nabla-\frac{e}{c} \boldsymbol{A}\right)^{2}, \quad \boldsymbol{A}=(0, H x), \\
& H \phi=-\frac{\hbar^{2}}{2 m} \frac{\partial^{2} \phi}{\partial x^{2}}+\frac{1}{2 m}\left(p_{y}-\frac{e}{c} H x\right)^{2} \phi=\epsilon \phi, \text { where } \psi=\exp \frac{\mathrm{i} p_{y} y}{\hbar} \phi(x)
\end{aligned}
$$

with energy eigenvalues

$$
\epsilon=\hbar \omega\left(n+\frac{1}{2}\right), \quad \omega=\frac{e H}{m c} .
$$

The degeneracy of these energy levels, called Landau levels, equals $S e H / h c$, where $S$ is the area a vailable for the electron. Hence, the filling factor for an $N$ electron system is $\nu=N /$ degeneracy $=h c \rho / e H$, where $\rho$ is the density of electrons. The integer values of $\nu$ correspond to the so-called integer quantum IIall effect, while the fractional quantum Hall effect corresponds to $\nu=1 / 3,1 / 5,2 / 5, \ldots$, or more generally, to (cf. [3])

$$
\nu=\frac{1}{p+\frac{a_{1}}{p_{1}+\frac{a_{2}}{p_{2}+\ldots}}}
$$

where $a_{i}=0, \pm 1, p$ - odd integer, $p_{i}$ - even integer.

The problem of an electron in a magnetic field can also be considered in a cylindrical gauge (connected with an appropriate boundary symmetry). The eigenfunctions and energy eigenvalues have the form

$$
\phi_{n, m}(r, \varphi)=C_{n, m}\left(\frac{r}{l}\right)^{|m|} \exp \left(-\mathrm{i} m \varphi-\frac{r^{2}}{4 l^{2}}\right) L_{n}^{|m|}\left(\frac{r^{2}}{2 l^{2}}\right),
$$

$2 \pi l^{2}=h c / e H, E_{n, m}=\hbar \omega[n+(|m|-m+1) / 2]$, where $L_{n}^{|m|}$ are tlie Laguerre polynomials. The lowest Landau level is described by the functions for $n=0$, $m=0,1,2, \ldots$, i.e.

$$
\phi_{m}=\left(\frac{1}{2 \pi l^{2} 2^{m} m !}\right)^{1 / 2}\left(\frac{z}{l}\right)^{m} \exp \left(-\frac{|z|^{2}}{4 l^{2}}\right), \quad z=r \mathrm{e}^{\mathrm{i} \varphi} .
$$


Due to the above form of the one-particle function, the $N$ non-interacting particle system (with $N$ not exceeding the degeneracy of the Landau level) is described by a completely antisymmetric, homogeneous polynomial multiplied by the factor $\exp \left[-\sum_{j=1}^{N}\left|z_{j}\right|^{2} / 4 l^{2}\right]$. In a standard manner the appropriate wave functions are given by the Slater determinant, which (cf. Eq. (2.6)) reduces to Vandermonde determinant

$$
\left|\begin{array}{lllll}
1, & z_{1}, & z_{1}^{2}, & \ldots, & z_{1}^{N-1} \\
\ldots & \ldots & \ldots & \ldots & \ldots \\
1, & z_{N}, & z_{N}^{2}, & \ldots, & z_{N}^{N-1}
\end{array}\right|=\prod_{j<k}^{N}\left(z_{j}-z_{k}\right),
$$

i.e. the $N$-particle wave function has the form

$$
\phi\left(z_{1}, \ldots z_{N}\right)=c \prod_{j<k}^{N}\left(z_{j}-z_{k}\right) \exp \left(-\sum_{j}^{N} \frac{\left|z_{j}\right|^{2}}{4 l^{2}}\right) .
$$

The idea of Laughlin ([12]), crucial for understanding the fractional Hall effect, consists in introducing a small modification to the above formula, i.e.

$$
\phi_{p}\left(z_{1}, \ldots z_{N}\right)=c \prod_{j<k}^{N}\left(z_{j}-z_{k}\right)^{p} \exp \left(-\sum_{j}^{N} \frac{\left|z_{j}\right|^{2}}{4 l^{2}}\right)
$$

with $p=1,3,5 \ldots$ Observing ([3]) that

$$
\left|\phi_{p}\right|^{2}=c \exp \left[-\beta\left(2 p^{2} \sum_{j<k} \ln \left|z_{j}-z_{k}\right|+\frac{p}{2} \sum_{j} \frac{\left|z_{j}\right|^{2}}{l^{2}}\right)\right]
$$

is exactly the Boltzmann distribution (with $\beta=1 / p$ ) for $2 \mathrm{D}$ plasma and via direct interpretation ([12]) of equilibrium properties of plasma one can find that $p$ th Laughlin function describes the fractionally occupied lowest Landau level with the filling factor $1 / p$. The existence of the hierarchy of filling factors can be explained by introduction of quasi-holes and quasi-particles - ground Laughlin state excitations $([3,13])$.

The most important feature of the Laughlin state is that it is not a combination of one-particle states (with exception of $p=1$ case when the Laughlin function is the Slater function) and it even does not describe usual fermions. We rather describe some kind of superfermions ([14]) which topological distinctions are indicated in Fig. 1.

Recently $([14,15])$ the simple interpretation of fractional IIall states has been given. If we start with $n$ completely filled Landau levels in the external magnetic field $B$ and we attach additionally to each electron $2 m$ quanta of flux then we deal with a final mean effective magnetic field $B^{m}=( \pm 2 m+1) B$. This field changes the degeneracy of the Landau levels and the filling factor. A new filling fraction in effective field $B^{m}$ is ([15])

$$
\nu=\frac{n}{2 m n \pm 1} \text {. }
$$

For the simplest case of the initially filled lowest Landau level only (i.e. $n=1$ ) we find

$$
\nu=\frac{1}{2 m \pm 1} \text {. }
$$



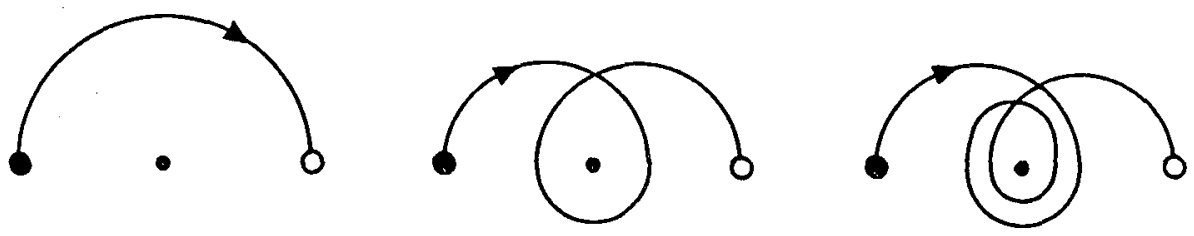

Fig. 1. Various types of topologically non-equivalent realizations of particle interchange on the plane corresponding to superfermions.

Both of the above formulae are sufficient to interpret the experimentally observed hierarchy of the fractional IIall states (the most prominently, for $n=1$ ).

\section{Braid group}

If one tries to construct a quantum theory for a classical system, one has to define the complex wave function $\Psi(x)$ determined on the classical configuration space $A$ of the considered system. In general this wave function can be multivalued $\Psi_{m}, m=1, \ldots C$. The only restriction is that when $x$ is taken along a closed loop in $A, \Psi_{m}$ must transform according to $C$-dimensional unitary representation of the first homotopy group of $A$, i.e. $\pi_{1}(A)$. Since this representation could in general be non-unique, therefore one can deal with distinct realizations of the quantum theory for the same classical system.

If we consider the $N$ identical particle system, then

$$
A=\left(M^{N} \backslash Z\right) / S_{N}
$$

where $M$ is the physically defined manifold for one particle, $M^{N}$ is the $N$ th Cartesian product, $Z$ is the subset of all elements of $M^{N}$ that two or more particle coordinates coincide, $S_{N}$ is the permutation group of $N$ elements.

If $M=R^{3}$ (as for $3 \mathrm{D}$ particles), then

$$
\pi_{1}(A)=S_{N}
$$

and we deal only with two distinct one-dimensional unitary irreducible representations of $S_{N}: 1$ - corresponding to the quantum theory of bosons, and \pm 1 (according to the sign of permutation) - corresponding to the theory of fermions.

If, however, $M=R^{2}$ then the situation is much more complicated. For 2D particles in the plane $([16])$

$$
\pi_{1}(A)=B_{N}
$$

where $B_{N}$ is the so-called braid group. The structure of this group is as follows:

$$
\begin{aligned}
& B_{N}=\left\{a_{1}, \ldots, a_{N-1} \mid a_{i} a_{i+1} a_{i}=a_{i+1} a_{i} a_{i+1}\right. \\
& \left.a_{i} a_{j}=a_{j} a_{i} \text { for }|i-j| \geq 2\right\}
\end{aligned}
$$

where $a_{i}$ interchanges particles $i$ and $i+1$. In order to compare $B_{N}$ with the $S_{N}$ group let us also present the definition of $S_{N}$ 


$$
\begin{gathered}
S_{N}=\left\{a_{1}, \ldots, a_{N-1} \mid a_{i}^{2}=e ; a_{i} a_{i+1} a_{i}=a_{i+1} a_{i} a_{i+1}\right. \\
\left.a_{i} a_{j}=a_{j} a_{i} \text { for }|i-j| \geq 2\right\} .
\end{gathered}
$$

From the above it is clear that $B_{N}$ is an infinite group, without cyclic elements. $S_{N}$ is not a subgroup of $B_{N}\left(S_{N}\right.$ is the quotient group, $S_{N}=B_{N} /$ \{minimal normal subgroup of elements $a_{i}^{2}$ for all $\left.\left.i\right\}\right)$. Hence, each representation of $S_{N}$ is the representation of $B_{N}$ but not conversely.

One-dimensional unitary representations of $B_{N}$ are

$\mathrm{e}^{\mathrm{i} \Theta}$

where $\Theta=0$ corresponds to bosons, $\Theta=\pi$ to fermions and other values of $\Theta$ correspond to anyons - new types of quantum particles in the plane.

It is interesting to note that $\pi_{1}(A)$ for other manifolds like a torus (relating to periodic boundary conditions in the plane) or a sphere admits also the existence of anyons ([17]). It might be important with regard to superconductivity of fullerites, where multiply connected topology could be related to charge conducting particles.

\section{Chern-Simons term}

Due to the Aharonov-Bohm effect it is clear that for 2D particles with a charge $Q$ and a flux $\Phi$ attached to each particle, the wave function acquires the phase

$$
\mathrm{e}^{\mathrm{i} Q \oint \boldsymbol{A} \cdot \mathrm{d} \boldsymbol{l}}=\mathrm{e}^{\mathrm{i} 2 Q \boldsymbol{\Phi}},
$$

if one particle turns the loop around the other. So, the statistics transmutation caused by the flux $\Phi$ is $\Delta \Theta=Q \Phi$.

The question is how to couple the flux to the charge of the particle. In the formal manner it is done by inclusion of the so-called Chern-Simons term to effective Lagrangian of the system. This term has the structure $([7,8,18])$

$$
\Delta L=e j^{\nu} A_{\nu}-\frac{\mu}{2} \epsilon^{\alpha \eta \nu} A_{\alpha} \partial_{\eta} A_{\nu} .
$$

The dynamics of the gauge field $A$ is described by the equation

$$
e j^{\nu}=\frac{\mu}{2} \epsilon^{\nu \alpha \beta} \partial_{\alpha} A_{\beta} \text {. }
$$

From the above equation it follows immediately that

$$
\int j^{0} \mathrm{~d} s=\int \frac{\mu}{2} \epsilon^{0 \alpha \beta} \partial_{\alpha} A_{\beta} \mathrm{d} s=\mu \Phi=Q=N e .
$$

One can rewrite the above within a more complete formula for the full Lagrangian of non-interacting particles

$$
L=\sum_{i=1}^{N}\left\{\frac{m}{2} \dot{x}_{i}^{2}+e\left[-A^{0}\left(x_{i}\right)+x_{i} \cdot A\left(x_{i}\right)\right]\right\}+\frac{\mu}{2} \int \mathrm{d}^{2} x \epsilon^{\rho \sigma \nu} A_{\rho} \partial_{\sigma} A_{\nu},
$$

where $x_{i}$ are the particle coordinates, $\boldsymbol{A}$ is the vector part of a field $A$, and $e j^{\rho}=\frac{\mu}{2} \epsilon^{\rho \tau \sigma} \partial_{\tau} A_{\sigma}, e j^{0}=\mu \epsilon_{\alpha \beta} \partial_{\alpha} A_{\beta}$. Hence, the transmutation: $\Delta \Theta=e^{2} / 2 \mu$ (the additional $1 / 2$ factor is caused by pure gauge field in term $\mathrm{e}^{\mathrm{i} \int L}-\mathrm{cf}$. [19]). 
If we choose the specific gauge $\partial_{\alpha} A_{\alpha}=0$, we find (by virtue of dynamics equation)

$$
A_{\alpha}(x)=\frac{e}{2 \pi \mu} \sum_{i=1}^{N} \epsilon_{\alpha \beta} \frac{\left(x-x_{i}\right)_{\beta}}{\left|x-x_{i}\right|^{2}},
$$

which allows us to write the Hamiltonian (corresponding to the Lagrangian (4.5)) in the following form ([18]):

$$
H=\frac{1}{2 m} \sum_{i=1}^{N}\left[p_{i}-e \boldsymbol{A}\left(x_{i}\right)\right]^{2} .
$$

This Hamiltonian is the startpoint for all further considerations of the anyon system. Let us underline, however, that the above formulation is given in the fermion representation of anyons, since the initial Lagrangian (4.5) was written for spinless 2D fermions with the flux attached (by the Ch.-S. term). Note also that in $2 \mathrm{D}$ we have no usual quantization of the spin since in the plane all revolutions commute in contrary to the $3 \mathrm{D}$ case.

\section{Anyon superconductivity}

If one considers the system of $N$ non-interacting anyons in the anyon representation, the relevant Hamiltonian and translation operators have the simple form $(\hbar=c=1)$

$$
H=-\frac{1}{2 m} \sum_{i=1}^{N} \frac{\mathrm{d}^{2}}{\mathrm{~d}\left(x_{i}^{\alpha}\right)^{2}}, \quad p_{\alpha}=-\sum_{i=1}^{N} \mathrm{i} \frac{\mathrm{d}}{\mathrm{dx}_{\mathrm{i}}^{\alpha}} .
$$

In this representation the wave function is, however, very inconvenient. It is multivalued owing to the phase factor $\mathrm{e}^{\mathrm{i} \Theta}$ due to interchange of identical particles.

In order to simplify the wave function one can perform the transmutation to bosons or fermions by adding an appropriate flux to each particle so that the Aharonov-Bohm phase gives the phase $\Theta$. Hence, the anyons of the $\Theta$ type (with a charge $q$ ) can be represented as fermions with the flux $\phi$ that $q \phi / 2=\theta-\pi$, or bosons with the flux $\varphi$ that $q \varphi / 2=\Theta$. After the transmutation to fermion representation (for $\Theta=\pi(1-1 / f)$ ) we have

$$
H=-\frac{1}{2 m} \sum_{i=1}^{N} \frac{\mathrm{D}^{2}}{\mathrm{D}\left(x_{i}^{\alpha}\right)^{2}}, \quad P_{\alpha}=-\mathrm{i} \sum_{i=1}^{N} \frac{\mathrm{D}}{\mathrm{Dx}_{\mathrm{i}}^{\alpha}},
$$

where

$$
\frac{\mathrm{D}}{\mathrm{Dx}_{\mathrm{i}}^{\alpha}}=\frac{\mathrm{d}}{\mathrm{dx}_{\mathrm{i}}^{\alpha}}+\mathrm{i} e A_{i}^{\alpha}, \quad A_{i}^{\alpha}=\frac{1}{e f} \sum_{j \neq i} \frac{\epsilon_{\alpha \beta}\left(x_{i}^{\beta}-x_{j}^{\beta}\right)}{\left|x_{i}-x_{j}\right|^{2}} .
$$

Note, however, that in the anyon representation

$$
\left[H, p_{\alpha}\right]=0, \quad\left[p_{\alpha}, p_{\beta}\right]=0 .
$$

In the fermion representation we deal with the same property

$$
\left[H, P_{\alpha}\right]=0, \quad\left[P_{\alpha}, P_{\beta}\right]=0 .
$$


The basic idea of anyon superconductivity consists in the conjecture ([20]) that the phase transition to superconducting state is described in the anyon system by spontaneous violation of the commutation relation according to the following formula:

$$
\left[P_{\alpha}, P_{\beta}\right]=\mathrm{i} \epsilon_{\alpha \beta} B N,
$$

with $B$ being the macroscopic order parameter ( $N$ is the number of particles). The above holds only for statistics parameters $\Theta=\pi(1-1 / f)$ with $f$ being an integer (which will be commented afterwards) and for other statistics superconductivity it does not appear.

Because the idea of the violation of a commutation relation is the generalization of the spontaneous symmetry violation, an analogue of the Goldstone theorem is suggested (cf. [20]). The appropriate massless boson is the gapless collective (sound-like) mode which was found for non-interacting anyon system by Fetter, IIanna and Laughlin ([21]).

There exists a simple model of the realization of the described above violation conjecture. It is the so-called mean field approximation. Within this approach the flux sum (in the fermion representation) is represented by the flux of the mean statistical field (see Appendix A)

$$
\begin{aligned}
A_{i}^{\alpha} & =\frac{1}{e f} \sum_{j \neq i} \frac{\epsilon_{\alpha \beta}\left(x_{i}^{\beta}-x_{j}^{\beta}\right)}{\left|x_{i}-x_{j}\right|^{2}}=\frac{1}{e f} \int \mathrm{d}^{2} y \frac{\epsilon_{\alpha \beta}\left(x_{i}^{\beta}-y^{\beta}\right)}{\left|x_{i}-y\right|^{2}} j^{0}(y) \\
& \simeq \frac{1}{2} B \widehat{z} \times r,
\end{aligned}
$$

if we put $j^{0}=$ const $=\rho, B=2 \pi \rho / e f$.

Note that the better approximation, the higher density $\rho$ is. In the presence of the mean field $B$

$$
\begin{aligned}
& H=-\frac{1}{2 m} \sum_{k} \frac{\mathrm{D}^{(0) 2}}{\mathrm{D}\left(x_{k}^{\alpha}\right)^{2}}, \quad \frac{\mathrm{D}^{(0)}}{\mathrm{D} x_{k}^{\alpha}}=\frac{\mathrm{d}}{\mathrm{d} x_{k}^{\alpha}}+\mathrm{i} A_{k}^{(0) \alpha}, \\
& P_{\alpha}=-\mathrm{i} \sum_{k} \frac{\mathrm{D}^{(0)}}{\mathrm{D} x_{k}^{\alpha}}+B \epsilon_{\alpha \beta} x^{\beta}, \quad \boldsymbol{B}=\operatorname{rot} A^{(0)}, \\
& {\left[P_{\alpha}, P_{\beta}\right]=\mathrm{i} \epsilon_{\alpha \beta} N B, \quad\left[H, P_{\alpha}\right]=0 .}
\end{aligned}
$$

For this mean field $B$ the degeneracy of the Landau levels is $S e B / 2 \pi$ and the filling factor is $(1-\Theta / \pi)^{-1}$. If $\Theta=\pi(1-1 / f), f$ being an integer, one obtains $f$ completely filled Landau levels. Hence, only for such statistics parameter valucs we deal with the gap in the energy spectrum. Let us underline that this model yields the following superconducting properties:

1. an energy gap $\Delta=e B / m$,

2. the Meissner effect (for the ground state of non-interacting anyon gas),

3. a gapless collective excitation (sound-like boson mode - the Goldstone mode which restitutes the commutation relation) $\omega=\sqrt{f} \omega_{\mathrm{c}} a_{0} q$, where $\omega_{\mathrm{c}}=e B / m, a_{0}=[1 / e B]^{1 / 2}$ (both the Hartree-Fock approximation [22] and the random-phase approximation (RPA) [21] support this result). 
To be more specific let us comment shortly on the Meissner effect within the mean field approach. If $b$ is the external magnetic field, the total magnetic field $B+b$ (for $b \uparrow \uparrow B$ ) or $B-b$ (for $b \uparrow \downarrow B$ ) causes the rearrangement of the Landau levels which leads to increase in energy according to the following formulae:

$$
\begin{aligned}
& E=\frac{f^{2} e^{2}}{4 \pi m}\left[B^{2}+\frac{1}{f} b B-\left(1-\frac{1}{f}\right) b^{2}\right] \quad \text { for } \quad b \uparrow \uparrow B, \\
& E=\frac{f^{2} e^{2}}{4 \pi m}\left[B^{2}+\frac{1}{f} b B-\left(1+\frac{1}{f}\right) b^{2}\right] \quad \text { for } \quad b \uparrow \downarrow B .
\end{aligned}
$$

It is interesting to observe that the penetration of the external magnetic field is inconvenient for the sufficiently low field $b$, despite its orientation with respect to the internal statistical mean field $B$ (linear terms in Eqs. (5.11) and (5.12)). Nevertheless, for higher $b$, with the nonlinear terms being dominant, the $b$ field penetration reduces the energy distinctly for two respective orientations of $B$ and $b$. The critical values of $b$ are

$$
\begin{aligned}
& b_{\mathrm{c}}^{\uparrow \uparrow}=\frac{\pi \rho}{e f^{2}}\left(1-\frac{1}{f}\right)^{-1}, \\
& b_{\mathrm{c}}^{\uparrow \downarrow}=\frac{\pi \rho}{e f^{2}}\left(1+\frac{1}{f}\right)^{-1} .
\end{aligned}
$$

Description of the Meissner effect (in the framework of the random-phase approximation [20,21]) allows also for estimation of the coherence length for the anyon superconductor via formal application of the Pippard formula to the correlation function for the anyon gas. This function has the following form (for $f=2$, cf. [21]): $K(q)=K_{y y}(q, \omega=0)=\rho\left[1-\left(q a_{0}\right)^{2} 3 / 8+\ldots\right]$ and via the comparison with the Pippard formula $\rho\left[1-\left(q \xi_{0}\right)^{2} 1 / 5+\ldots \ldots\right]$ one can find that $\xi_{0}=(15 / 8)^{1 / 2} a_{0}$ ( $\xi_{0}$ turns out to be of order of the interparticle separation length).

\section{Linear response theory for ideal anyon gas}

The free-anyon Ilamiltonian $H$ can be separated into two parts: $I=I_{0}+$ $H_{1}$, where $H_{0}$ is the mean field Hamiltonian which can be treated as the unperturbed term and

$$
H_{1}=\sum_{j} \frac{e}{c m}\left[\left(\boldsymbol{p}_{j}+\frac{e}{c} \overline{\boldsymbol{A}}_{j}\right)\left(\overline{\boldsymbol{A}}_{j}-\boldsymbol{A}_{j}\right)+\frac{e}{2 c}\left|\boldsymbol{A}_{j}-\overline{\boldsymbol{A}}_{j}\right|^{2}\right]
$$

is the interaction Hamiltonian $([18,21])$. In the above formula $\overline{\boldsymbol{A}}_{j}$ is the averaged statistical field (mean field) and $\boldsymbol{A}_{j}=\boldsymbol{A}\left(x_{j}\right)$ is its exact value. The mean field current density is given by

$$
j(r)=\frac{1}{2 m} \sum_{j}\left\{p_{j}+\frac{e}{c} \bar{A}_{j}, \delta\left(r-r_{j}\right)\right\}
$$

(braces denote an anticommutator) and the full current density is

$$
J(r)=\frac{1}{2 m} \sum_{j}\left\{p_{j}+\frac{e}{c} A_{j}, \delta\left(r-r_{j}\right)\right\},
$$


where $j, J$ are the vector parts of $j^{\mu}, J^{\mu}$, respectively, with $\mu=0, x, y$. Let us define $j^{0}, J^{0}$ as density fluctuations

$$
j^{0}=J^{0}=\sum_{j} \delta\left(\boldsymbol{r}-\boldsymbol{r}_{j}\right)-\rho=\tilde{\rho} .
$$

The problem of interest is the linear response of the system to an external electromagnetic field described by the scalar potential $\Phi^{\mathrm{ex}}$ and vector potential $\boldsymbol{A}^{\mathrm{ex}}$. The relevant external perturbation Hamiltonian is given by the formula

$$
H^{\mathrm{ex}}(t)=-e \int \mathrm{d} \boldsymbol{r}\left[J^{0}(r, t) \Phi^{\mathrm{ex}}(r, t)-\frac{1}{c} J(r, t) A^{\mathrm{ex}}(r, t)\right] .
$$

The linear response contains a diamagnetic and a paramagnetic contributions. The second one is proportional to the retarded current-current correlation function

$$
\Delta_{R}^{\mu \nu}\left(\boldsymbol{r} t, \boldsymbol{r}^{\prime} t^{\prime}\right)=-\mathrm{i} \theta\left(t-t^{\prime}\right)\left\langle\left[J^{\mu}(\boldsymbol{r} t), J^{\nu}\left(\boldsymbol{r}^{\prime} t^{\prime}\right)\right]\right\rangle
$$

It is convenient to represent the explicit form of the response in the momentum space. Then the induced electromagnetic current equals

$$
-e\left\langle J^{\mu}(q, \omega)\right\rangle=-\frac{c}{\pi}\left[K^{\mu k}(q, \omega) A_{k}^{\mathrm{ex}}(\boldsymbol{q}, \omega)-K^{\mu 0}(q, \omega) \Phi^{\mathrm{ex}}(\boldsymbol{q}, \omega)\right] .
$$

The kernel $K^{\mu \nu}$ characterizes the linear response of the system and is equal to

$$
K^{\mu \nu}(q, \omega)=\frac{4 \pi \bar{\rho} e^{2}}{m c^{2}} \delta^{\mu \nu}\left(1-\delta^{\mu 0}\right)+\frac{4 \pi e^{2}}{\hbar c^{2}} \Delta_{R}^{\mu \nu}(q, \omega) .
$$

IIence, it is clear that in order to get the linear response it is necessary to calculate the full current operator retarded correlation function $\Delta_{R}^{\mu \nu}$. However, it is convenient to consider first the retarded correlation function of two average-field currents (i.e. for $j$ instead of $J$ )

$$
D_{R}^{\mu \nu}\left(r t, r^{\prime} t^{\prime}\right)=-\mathrm{i} \theta\left(t-t^{\prime}\right)\left\langle\left[j^{\mu}(r t), j^{\nu}\left(r^{\prime} t^{\prime}\right)\right]\right\rangle \text {. }
$$

The random-phase approximation consists in approaching $D_{R}$ by the sum of bubble graphs which can be written as

$$
D_{R}^{\mathrm{RPA}}(\boldsymbol{q}, \omega)=\left[I-D_{R}^{0}(\boldsymbol{q}, \omega) V(q)\right]^{-1} D_{R}^{0}(q, \omega) .
$$

The interaction matrix $V$ can be found if the Hamiltonian $H_{1}$ is approximated by the quadratic form in the momentum space

$$
H_{1}^{\mathrm{RPA}}=\frac{1}{2} S^{-1} \sum_{q \neq 0} V^{\mu \nu}(q) j_{q}^{\nu} j_{-q}^{\mu} .
$$

Then the matrix $V^{\mu \nu}(q)$ has the form (we assume $\omega_{c}$ and $a_{0}$ to be frequency and length units, respectively)

$$
V(q)=\frac{2 \pi}{f q^{2}}\left(\begin{array}{ccc}
1 & -\mathrm{i} q_{y} & \mathrm{i} q_{x} \\
\mathrm{i} q_{y} & 0 & 0 \\
-\mathrm{i} q_{x} & 0 & 0
\end{array}\right)
$$

The self-consistent collective density modes of the system (which remains when $A^{\text {ex }}=0$ ) are obtained from the zeros of the determinant

$$
\operatorname{det}\left[I-D_{R}^{0}(\boldsymbol{q}, \boldsymbol{\omega}) V(\boldsymbol{q})\right]=0 .
$$


As usual, $D_{R}^{0}$ can be found via analytic continuation of the appropriate time-ordered correlation function.

The procedure described above was performed by Fetter, Hanna and Laughlin within $T=0$ formalism ([21, 23]). The most important results are: the collective mode $\omega_{q} \simeq \sqrt{f} q$ and Meissner effect $K^{\mathrm{RPA}}(q) \simeq 1-f q^{2} 3 / 16$, both being a strong support for the idea of superconductivity of the ideal anyon gas with $\Theta=\pi(1-1 / f), f$ being an integer ([24]). In the following section we generalize the RPA to the non-zero temperature case.

\section{Anyon gas at non-zero temperature random-phase approximation}

A very interesting problem is to generalize the anyon RPA for non-zero temperatures. The linear response at finite temperatures is described as in the previous section if, however, brackets $(.$.$) denote thermodynamic expectation$ values (i.e. grand canonical ensemble averaging). The problem is to calculate the temperature particle and current density correlation function which can be written as

$$
D^{0}(1,2)=-P^{\mu}\left(1,1^{\prime}\right) P^{\nu}\left(2,2^{\prime}\right)\left\langle T\left[\hat{\Psi}^{+}\left(1^{\prime}\right) \hat{\Psi}(1) \hat{\Psi}^{+}\left(2^{\prime}\right) \hat{\Psi}(2)\right]\right\rangle_{0},
$$

where $\langle\ldots\rangle_{0}$ is averaging with $\mathrm{e}^{\left(H_{0}-\mu N\right) / k T}$ and

$$
P\left(1,1^{\prime}\right)=\frac{1}{2}\left[\frac{1}{\mathrm{i}}\left(\nabla_{1}-\nabla_{1^{\prime}}\right)+\widehat{z} \times r_{1}\right], \quad P^{0}=1 .
$$

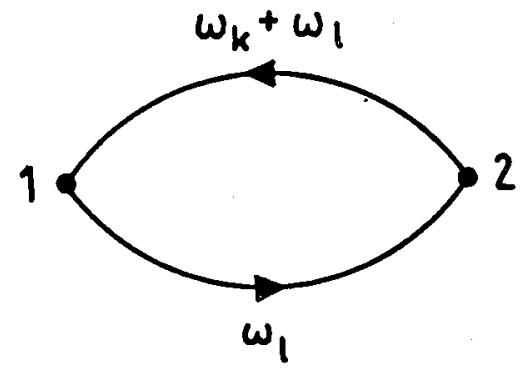

Fig. 2. The Feynman diagram for the non-zero part of the correlation function given by Eq. (7.1).

In Eq. (7.1) we have omitted the terms associated with the uniform density in Eq. (6.4). From the Wick theorem we obtain two contractions but only the one presented in Fig. 2 contributes. The other vanishes because for $\nu, \mu=0$ the appropriate matrix elements cancel with those omitted in Eq. (7.1) and for $\nu, \mu \neq 0$ the current vanishes in the unperturbed state. By virtue of the Feynman rules for the temperature Green functions one can write 


$$
D_{\mu \nu}^{0}\left(r_{1}, r_{2}, u_{k}\right)=\frac{1}{\beta} \sum_{w_{l}} P^{\mu}\left(1,1^{\prime}\right) P^{\nu}\left(2,2^{\prime}\right) G_{0}\left(r_{1}, r_{2}^{\prime}, \omega_{l}+u_{k}\right) G_{0}\left(r_{2}, r_{1}^{\prime}, \omega_{l}\right),
$$

where $\beta=\hbar / k T$ and $\omega_{l}=(2 l+1) \pi / \beta$ since we deal with the fermion representation of the anyon gas, and $u_{k}=2 k \pi / \beta$ (being the transfer of the Matsubara frequency for the fermion system). The Matsubara-Green function has the form

$$
G_{0}\left(r_{1}, r_{2}^{\prime}, \omega_{l}\right)=\sum_{\epsilon_{m}} \frac{\Pi_{m}\left(r_{1}, r_{2}^{\prime}\right)}{\mathrm{i} \omega_{l}-\left(\epsilon_{m}-\mu / \hbar\right)},
$$

where $I_{m}$ is the projector on the $m$ th Landau level $\left(\epsilon_{m}=m+1 / 2\right.$, in our units). Similarly as in [24] the Fourier transform of $D^{0}$ can be presented as (see Appendix B)

$$
D_{\mu \nu}^{0}\left(q, u_{k}\right)=\sum_{m n} d_{m n}\left(u_{k}\right) \int \frac{\mathrm{d} p}{(2 \pi)^{2}} P^{\mu} P^{\nu *} g_{m}\left(p+\frac{1}{2} q\right) g_{n}\left(p-\frac{1}{2} q\right)
$$

(functions $g_{n}$ are defined in Appendix B), where $\boldsymbol{P}=\boldsymbol{p}+\frac{1}{2} \mathrm{i} \hat{z} \times \nabla_{q}$ and $P^{0}=1$ and

$$
\begin{aligned}
& d_{m n}\left(u_{k}\right)=\frac{1}{\beta} \sum_{\omega_{l}} \frac{1}{\left[\mathrm{i} \omega_{l}+\mathrm{i} u_{k}-\left(\epsilon_{m}-\mu / \hbar\right)\right]} \frac{1}{\left[\mathrm{i} \omega_{l}-\left(\epsilon_{n}-\mu / \hbar\right)\right]} \\
& \quad=\frac{n_{n}^{0}-n_{m}^{0}}{\mathrm{i} u_{k}-(m-n)}
\end{aligned}
$$

if $u_{k} \neq 0, n_{m}^{0}=\left[\exp \left(\epsilon_{m}-\mu / \hbar\right) \beta+1\right]^{-1}$. However, if $u_{k}=0$ and $m=n$ we deal with a double pole in Eq. (7.6) and thus $d_{n n}\left(u_{k}=0\right)=-\beta n_{n}^{0}\left(1-n_{n}^{0}\right)$. After some calculation (see Appendix B) and the analytic continuation (i $u_{k} \rightarrow \omega$ ) one finds

$$
D_{R}^{0}(q, \omega)=\frac{f}{2 \pi}\left(\begin{array}{ccc}
q^{2} \Sigma_{0} & q \omega \Sigma_{0} & -\mathrm{i} q \Sigma_{1} \\
q \omega \Sigma_{0} & \omega^{2} \Sigma_{0}-1 & -\mathrm{i} \omega \Sigma_{1} \\
\mathrm{i} q \Sigma_{1} & \mathrm{i} \omega \Sigma_{1} & \Sigma_{2}
\end{array}\right)
$$

with $(\omega \neq 0)$

$$
\begin{aligned}
\Sigma_{j}= & \frac{\mathrm{e}^{-x}}{f} \sum_{m=1}^{\infty} \sum_{n=0}^{m-1}\left(n_{n}^{0}-n_{m}^{0}\right) \frac{m-n}{\omega^{2}-(m-n)^{2}} \frac{n !}{m !} x^{m-n-1}\left[L_{n}^{m-n}(x)\right]^{2-j} \\
& \times\left[(m-n-x) L_{n}^{m-n}(x)+2 x \frac{\mathrm{d} L_{n}^{m-n}(x)}{\mathrm{d} x}\right]^{j}
\end{aligned}
$$

where $x=|q|^{2} / 2, q=q \widehat{x}$.

For $\omega=0$ one finds the additional term

$$
\Sigma_{j}(\omega=0)=\Sigma_{j}(\omega \rightarrow 0)-\frac{\beta \mathrm{e}^{-x}}{2 f x} \sum_{n}\left[L_{n}(x)\right]^{2-j}\left[-x L_{n}(x)+2 x \frac{\mathrm{d} L_{n}(x)}{\mathrm{d} x}\right]^{j}
$$

Note that from the above, in the limit $T \rightarrow 0$ we rederive the result for $T=0$ (the additional term, for $\omega=0$, vanishes at $T=0$ ).

In order to find the collective density modes it is enough to know $\Sigma_{j}$ for small $\omega$ and $q$. The expansion with respect to $\omega$ and $q$ leads to the following expressions (for $\omega \neq 0$ ):

$$
\Sigma_{0} \simeq-1-\omega^{2}+\frac{3}{4} \alpha x
$$




$$
\begin{aligned}
& \Sigma_{1} \simeq-1-\omega^{2}+\frac{3}{2} \alpha x \\
& \Sigma_{2} \simeq-1-\omega^{2}+2 \alpha x
\end{aligned}
$$

where

$$
\alpha=2 \frac{1}{f} \sum_{m=0}^{\infty} n_{m}^{0}\left(m+\frac{1}{2}\right)
$$

in dimensionless units. Since the chemical potential is determined by the equation $\sum n_{n}^{0}=f$, one finds that $\mu / \hbar=f-1 / 2 \beta \exp (-f \beta)$ at low temperatures ([25]). Therefore $\alpha \simeq f+2 / f \exp (-\beta / 2)$ for $T \rightarrow 0$ (cf. also [26]).

Taking into account the above formulae we can rewrite the determinant (6.13) in the following form:

$$
D=\left(1+\Sigma_{1}\right)^{2}-\Sigma_{0}\left(1+\Sigma_{2}\right) \simeq-\omega^{2}+2 \alpha x .
$$

Hence, the collective mode has the sound-like spectrum

$$
\omega \simeq \sqrt{\alpha} q .
$$

To obtain the retarded correlation function $\Delta_{R}$ the difference between the true current and the mean-field current needs to be known. In the momentum space we obtain (cf. [24]):

$$
J_{q}-j_{q} \simeq \rho \boldsymbol{A}(\boldsymbol{q}) \tilde{\rho}_{q} .
$$

Therefore, one can write that $([21,23])$

$$
\Delta_{R} \simeq \Delta_{R}^{\mathrm{RPA}}=\left(I+U^{+}\right) D_{R}^{\mathrm{RPA}}(I+U),
$$

where $U$ is a simple matrix:

$$
U=\frac{\mathrm{i}}{q}\left(\begin{array}{lll}
0 & 0 & 1 \\
0 & 0 & 0 \\
0 & 0 & 0
\end{array}\right) .
$$

Hence, the linear response kernel is equal to

$$
K^{\mathrm{RPA}}=\frac{1}{D}\left(\begin{array}{ccc}
q^{2} \Sigma_{0} & q \omega \Sigma_{0} & -\mathrm{i} q\left(\Sigma_{s}-\Sigma_{0}\right) \\
q \omega \Sigma_{0} & \omega^{2} \Sigma_{0} & -\mathrm{i} \omega\left(\Sigma_{s}-\Sigma_{0}\right) \\
\mathrm{i} q\left(\Sigma_{s}-\Sigma_{0}\right) & i \omega\left(\Sigma_{s}-\Sigma_{0}\right) & 1+\Sigma_{2}
\end{array}\right)
$$

(taken in units of $4 \pi \bar{\rho} e^{2} / m c^{2}$ ) and

$$
\Sigma_{s}=\Sigma_{1}+\Sigma_{1}^{2}-\Sigma_{0} \Sigma_{2} .
$$

For $\omega=0$ one finds

$$
\begin{aligned}
& \Sigma_{0}(q, 0) \simeq-\frac{\tau_{0}}{q^{2}}-1+\frac{1}{2}\left(\tau_{0}+2 \tau_{1}\right)+\frac{1}{8} q^{2}\left[3 \alpha-\left(\tau_{0}+3 \tau_{1}+3 \tau_{2}\right)\right] \\
& \Sigma_{1}(q, 0) \simeq-1+\frac{1}{2}\left(\tau_{0}+2 \tau_{1}\right)+\frac{1}{4} q^{2}\left[3 \alpha-\left(\tau_{0}+3 \tau_{1}+3 \tau_{2}\right)\right] \\
& \Sigma_{2}(q, 0) \simeq-1+q^{2}\left[\alpha-\frac{1}{4}\left(\tau_{0}+4 \tau_{1}+4 \tau_{2}\right)\right]
\end{aligned}
$$


where $\tau_{j}=\beta / f \sum_{n=0}^{\infty} n^{j} n_{n}^{0}\left(1-n_{n}^{0}\right)$. Hence, the Meissner kernel $K(q)=K^{y y}(q, 0)$ is given by the formula

$$
K^{\mathrm{RPA}}(q)=\left.\frac{1+\Sigma_{2}}{D}\right|_{\omega=0} \simeq \frac{q^{2}}{\tau_{0}+q^{2}}
$$

and at low temperatures $\tau_{0} \simeq 2 \beta / f \exp (-\beta / 2)$ (cf. also [25, 26]).

Let us note that the same result was obtained by Fetter and Hannna within the Hartree approach ([26]). Since $K(0)=0$ the Meissner effect disappears at non-zero temperatures. In [26] it is argued, however, that for finite size sample the $q \rightarrow 0$ limit is, in fact, ranged by $1 / L$ quantity and the Meissner effect still persists at $T \neq 0$ though the screening of the field is only partial. Such an attitude allows one to predict the semi-critical temperature (via the condition $L \simeq a_{0} / \sqrt{\tau_{0}}, \mathrm{cf}$. [26]) at which the Meissner effect completely disappears.

The response kernel similar to that in Eq. (7.24) was also found by Hosotani, Hetrick and Lee within the self-consistent field approach ([27]). In that paper, however, the complete electrodynamics was taken into account which makes the physical situation quite distinct and the finite penetration (i.e., the Meissner effect) of the static magnetic field corresponds rather to the gap in collective excitation spectrum (cf. also [25]). Lack of the critical behaviour in Eq. (7.24) resembles a typical situation for various 2D systems for which no phase transition exists and other ideas instead of long range order are suggested, e.g. a Kosterlitz-Thouless phase transition. The relevance of the Kosterlitz-Thouless idea to anyon physics is discussed to some extent in [28].

\section{Comments}

A very interesting question is: are fractional statistics a vailable for one-dimensional systems? Despite the classic paper ([1]) where the pair of particles on the line was considered, the answer is not clear as yet owing to the peculiar topology of $1 \mathrm{D}$ (braidings correspond to the plane topology only). Also the permutation of particles on the line causes questions close to quantum theory interpretations (particles on the line are ordered and this influences the group structure).

Let us consider two non-interacting particles in 1D. Then the configuration space is the half plane and the IIamiltonian has the form

$$
H=-\frac{\hbar^{2}}{4 m} \frac{\partial^{2}}{\partial x^{2}}-\frac{\hbar^{2}}{m} \frac{\partial^{2}}{\partial z^{2}}
$$

where $x=\left(x_{1}+x_{2}\right) / 2$ and $z=\left|x_{1}-x_{2}\right| \geq 0$. Conservation of probability on the boundary of the configuration space (the line $x_{1}=x_{2}$ ) leads to the condition

$$
\left.\left[\Psi^{*}(x, z) \frac{\partial \Psi(x, z)}{\partial z}-\frac{\partial \Psi^{*}(x, z)}{\partial z} \Psi(x, z)\right]\right|_{z=0}=0
$$

(it means that the normal to boundary component of current vanishes). IIence [1],

$$
\left.\frac{\partial \Psi(x, z)}{\partial z}\right|_{z=0}=\eta \Psi(x, 0)
$$

and corresponding wave functions

$$
\Psi=\mathrm{e}^{\mathrm{i} \kappa x}\left(\cos k z+\frac{\eta}{k} \sin k z\right) .
$$


One can note that $\eta=0$ corresponds to bosons, while $\eta^{-1}=0$ to fermions. Other values of $\eta$ would be relevant to fractional statistics. The generalization of the above to $N$-particle system on the line is evident.

Let us note also that recently Haldane ([29]) has suggested the new definition of the fractional statistics free of the braiding term and thus applicable to 1D space. The spirit of this definition consists in the generalization of the Pauli principle. Limiting fractional statistics to topological excitations confined to the interior of condensed matter sample, the statistics is introduced by the $g$ factor in the following formula (cf. [29]):

$$
\Delta d_{\alpha}=-\sum_{\beta} g_{\alpha \beta} \Delta N_{\beta},
$$

which describes the available "free room" $-d_{\alpha}$ for particles of species $\alpha$ if the number of particles of species $\beta$ increases by $\Delta N_{\beta}$. For fermions $g_{\alpha \beta}=\delta_{\alpha \beta}$, while for bosons $g_{\alpha \beta}=0$. The above formulation leads to description of the IIall fluid equivalent with that of braiding group approach ([29]).

It is especially worth noting the fact that $g_{\alpha \beta}$ has to be a fractional number to preserve $d$ being an integer. Hence, if e.g., $g=1 / 3$ then $\Delta N=3 \cdot$ (integer number). It is the same property as for superconducting anyons with $\Theta=\pi(1-1 / f)$. In this case we have $f$ exactly filled Landau levels and we can change the number of particles only by the portion $\Delta N=f$. (integer number) since we have to add the same number of particles to each filled Landau level.

Since the integral for the average statistical field (see Appendix A) is convergent only for finite regions, it is not a coincidence that the Haldane idea of fractional statistics agrees with the mean field anyon treatment. To see this clearly, it is convenient to add some external flux to the "statistical" one (in the fermion representation) in order to locate all particles onto the lowest Landau level. Because of the energy gap we assume that particles do not achieve higher levels. The particle "free room" (in the lowest Landau level) is then given by $(\hbar=c=1$ )

$$
d_{\alpha}=\frac{\Phi^{\mathrm{ex}} e}{2 \pi}+\frac{(\pi-\Theta)}{\pi} N-(N-1)
$$

and by virtue of Eq. (8.5)

$$
g_{\alpha \alpha}=\frac{\Theta}{\pi}
$$

It is clear now that since the degeneracy is an integer, the number of particles in the system for e.g. $\Theta=\pi p / m(p / m-$ an irreducible fraction) can be changed only by portions of $m$. It is interesting to note that anyons on a sphere like those on a finite surface obey the same restriction.

\section{Acknowledgments}

The Authors would like to thank Andrzej Radosz for valuable comments and discussions. The work was supported by the project No. 202369101 of the Committee for Scientific Research. 


\section{Appendix A}

The mean field comes from the integral (5.7)

$$
\overline{\boldsymbol{A}}\left(\mathbf{r}^{\prime}\right)=\frac{1}{e f} \int \mathrm{d} \boldsymbol{r} \hat{z} \times \frac{r^{\prime}-r}{\left|r^{\prime}-r\right|^{2}} \rho(r)
$$

if we assume that $\rho(r)=$ const. This is the double integral for an infinite region $R^{2}$ and hence it is neccessary first to check its convergence. As it is known

$$
\int_{S} \int \psi \mathrm{d} s \text { is convergent } \Longleftrightarrow \int_{S} \int|\psi| \mathrm{d} s \text { is convergent. }
$$

However, in our case, the second integral is divergent for $R^{2}$ (and for any infinite region), then the integral given by Eq. (A.1)) is either.

But the integral (A.1) is convergent for finite regions. We have

$$
\bar{A}\left(\boldsymbol{r}^{\prime}\right)=\frac{1}{e f} \rho\left(\int_{D} \mathrm{~d} y \int \mathrm{d} x \frac{y^{\prime}-y}{\left|\boldsymbol{r}^{\prime}-\boldsymbol{r}\right|^{2}}, \int_{D} \mathrm{~d} x \int \mathrm{d} y \frac{x^{\prime}-x}{\left|\boldsymbol{r}^{\prime}-\boldsymbol{r}\right|^{2}}\right) .
$$

It should be noted that these integrals can be iterated only in the manner as it is written above (otherwise case one finds divergencies). Then

and

$$
\boldsymbol{B}=\nabla^{\prime} \times \overline{\boldsymbol{A}}\left(\boldsymbol{r}^{\prime}\right)=\left(\frac{\partial A_{y^{\prime}}}{\partial x^{\prime}}-\frac{\partial A_{x^{\prime}}}{\partial y^{\prime}}\right) \widehat{\boldsymbol{z}}
$$

$$
\begin{aligned}
B= & \frac{1}{e f} \rho\left(\int \mathrm{d} y \frac{\partial}{\partial y^{\prime}} \int \mathrm{d} x \frac{y^{\prime}-y}{\left|\boldsymbol{r}^{\prime}-\boldsymbol{r}\right|^{2}}-\int \mathrm{d} x \frac{\partial}{\partial x^{\prime}} \int \mathrm{d} y \frac{x^{\prime}-x}{\left|\boldsymbol{r}^{\prime}-\boldsymbol{r}\right|^{2}}\right) \\
& =\frac{1}{e f} \rho\left(\left.\int_{c}^{d} \mathrm{~d} y \frac{\boldsymbol{x}^{\prime}-x}{\left|\boldsymbol{r}^{\prime}-\boldsymbol{r}\right|^{2}}\right|_{x(y)} ^{X(y)}+\left.\int_{a}^{b} \mathrm{~d} x \frac{y^{\prime}-y}{\left|\boldsymbol{r}^{\prime}-\boldsymbol{r}\right|^{2}}\right|_{y(x)} ^{Y(x)}\right),
\end{aligned}
$$

where $a, b, c, d, x(y), X(y), y(x), Y(x)$ describe the limits of the region $D$. It can be seen that the above integral is equal to the contour integral

$$
B=\frac{1}{e f} \rho(-1)\left(\oint_{C} \frac{y^{\prime}-y}{\left|\boldsymbol{r}^{\prime}-\boldsymbol{r}\right|^{2}} \mathrm{~d} x+\frac{x^{\prime}-x}{\left|\boldsymbol{r}^{\prime}-\boldsymbol{r}\right|^{2}} \mathrm{~d} y\right),
$$

which is the Gauss integral and it equals

$$
B=\frac{1}{e f} \rho\left\{\begin{array}{ccl}
2 \pi & \text { if } & \left(x^{\prime}, y^{\prime}\right) \in D \\
0 & \text { otherwise }
\end{array}\right.
$$

Therefore the mean field is well defined for finite regions. However, one can obtain the mean field for $R^{2}$ while taking the limit of Eq. (A.7) as the support of $\rho$ extends over all the space (cf. [20]).

\section{Appendix B}

The Landau level projector can be written as follows ([23]):

$$
\Pi_{n}\left(r_{1}, r_{2}\right)=\exp \left(\frac{1}{2} \mathrm{i} \hat{z} \cdot r_{1} \times r_{2}\right) g_{n}\left(r_{1}-r_{2}\right),
$$

where

$$
g_{n}\left(r_{1}-r_{2}\right)=\frac{1}{2 \pi} L_{n}\left(\frac{|z|^{2}}{2}\right) \exp \left(-|z|^{2} / 4\right),
$$


$|z|=\left|r_{1}-r_{2}\right|$ and $L_{n}$ are the Laguerre functions. The corresponding Fourier transform is given by

$$
g_{n}(k)=(-1)^{n} 2 L_{n}\left(2|k|^{2}\right) \mathrm{e}^{-|k|^{2}} .
$$

To calculate the correlation function, Eq. (7.1), let us first consider the $k 0(k=x, y)$ element

$$
\begin{aligned}
D_{0}^{k 0}\left(r_{1}, r_{2}, u_{k}\right)= & \frac{1}{2}\left[\frac{1}{\mathrm{i}}\left(\nabla_{1}-\nabla_{1^{\prime}}\right)+\widehat{z} \times r_{1}\right]^{k} \exp \left[\frac{1}{2} \mathrm{i} \widehat{z}\left(r_{1} \times r_{2}+r_{2} \times r_{1}^{\prime}\right)\right] \\
& \times \sum_{m n} g_{m}\left(r_{1}-r_{2}\right) g_{n}\left(r_{2}-r_{1}\right) d_{m n}\left(u_{k}\right),
\end{aligned}
$$

where $d_{m n}$ is defined by Eq. (7.6). Introducing the spatial Fourier transform of the translationally invariant functions $g_{m}$ and $g_{n}$ we obtain

$$
\begin{aligned}
& D_{k 0}^{0}\left(r_{1}, r_{2}, u_{k}\right)=\sum_{m n} d_{m n}\left(u_{k}\right) \int \frac{\mathrm{d} p \mathrm{~d} \boldsymbol{q}}{(2 \pi)^{4}} g_{m}\left(p+\frac{q}{2}\right) g_{n}\left(p-\frac{q}{2}\right) \\
& \quad \times\left(p+\frac{1}{2} \widehat{z} \times r_{12}\right)^{k} \exp \left(\mathrm{i} \boldsymbol{q} \cdot \boldsymbol{r}_{12}\right) .
\end{aligned}
$$

The spatial factor $\widehat{z} \times \boldsymbol{r}_{12}$ can be written as $-\mathrm{i} \widehat{\boldsymbol{z}} \times \nabla_{q}$ acting on the exponential term $\exp \left(\mathrm{i} q \cdot r_{12}\right)$ and an integration by parts with respect to $q$ expresses the above equation as a spatial Fourier integral. Similarly Eq. (7.5) can be found. ([23])

Let us consider $u_{k} \neq 0$. In the element $D_{00}^{0}$ we are dealing with the integral

$$
I^{m n}=\int \frac{\mathrm{d} p}{(2 \pi)^{2}} g_{m}\left(p+\frac{q}{2}\right) g_{n}\left(p-\frac{q}{2}\right)=\frac{\mathrm{e}^{-x} x^{m-n} n !}{2 \pi m !}\left[L_{n}^{m-n}(x)\right]^{2},
$$

which is symmetric with respect to the interchange of indices. Therefore

$$
D_{00}^{0}=\sum_{m n} d_{m n} I^{m n}=\sum_{m>n} d_{m n} I^{m n}+\sum_{n>m} d_{m n} I^{m n}
$$

$\left(d_{m m}=0\right.$ for $\left.u_{k} \neq 0\right)$. After the interchange of indices in the second sum one finds

$$
D_{00}^{0}=\sum_{m>n} I^{m n}\left(d_{m n}+d_{n m}\right)=\frac{f}{2 \pi} q^{2} \Sigma_{0}
$$

The elements $k 0$ and $0 l$ involve the integrals

$$
I_{k}^{m n}=\int \frac{\mathrm{d} \boldsymbol{p}}{(2 \pi)^{2}} p_{k} g_{m}\left(p+\frac{q}{2}\right) g_{n}\left(p-\frac{q}{2}\right)
$$

and (cf. [23])

$$
I_{k}^{m n}=q_{k}(2 x)^{-1}(m-n) I^{m n} .
$$

Then

$$
D_{0 x}^{0}=D_{x 0}^{0}=\sum_{m n} d_{m n} I_{x}^{m n}=\sum_{m>n} I_{x}^{m n}\left(d_{m n}-d_{n m}\right)=\frac{f}{2 \pi} \mathrm{i} u_{k} q \Sigma_{0},
$$

which follows from the antisymmetry of $I_{x}^{m n}\left(\Sigma_{j}(\omega)=\Sigma_{j}\left(\mathrm{i} u_{k}\right)\right)$. Moreover,

$$
D_{0 y}^{0}=D_{y 0}^{0}=-\mathrm{i} q \frac{\partial}{\partial x}\left(I^{m n}\right)=-\frac{f}{2 \pi} \mathrm{i} q \Sigma_{1} .
$$


The other elements involve integrals $I_{k l}^{m n}$ which are symmetric under interchange $m \leftrightarrow n$. They have the form (cf. [23])

$$
\begin{aligned}
& I_{k l}^{m n}=\frac{e^{-x} x^{m-n-1}}{16 \pi m ! n !}\left\{\left[L_{m n}^{2} x^{-1}\left[2(m-n)^{2}-(m-n-x)\right]-\left(L_{m n}^{2}\right)^{\prime}\right\} q_{k} q_{l}\right. \\
& +\left\{L_{m n}^{2} x^{-1}(m-n-x)-\left(L_{m n}^{2}\right)^{\prime}+4 x\left[\left(L_{m n}^{\prime}\right)^{2}-L_{m n} L_{m n}^{\prime \prime}\right]\right\} \\
& \left.(\hat{z} \times q)_{k}(\widehat{z} \times q)_{l}\right],
\end{aligned}
$$

where $L_{m n}=L_{n}^{m-n}, m>n$. Moreover, the derivative term $\left(\hat{z} \times \nabla_{q}\right)_{k}\left(\hat{z} \times \nabla_{q}\right)_{l} I^{m n}$ which can be rewritten as $\partial\left[(\widehat{z} \times q){ }_{l} \partial_{x} I^{m n}\right] / \partial q_{k}$ gives two terms: one proportional to $\delta_{k l}$ and one proportional to $(\widehat{z} \times q)_{k}(z \times q)_{l}$. Therefore

$$
D_{x y}^{0}=-D_{y x}^{0}=\mathrm{i} \sum_{m>n}\left(d_{m n}-d_{n m}\right)(m-n) \frac{\partial}{\partial x} I^{m n}=-\frac{f}{2 \pi} u_{k} \Sigma_{1}
$$

and

$$
\begin{aligned}
D_{x x}^{0} & =\sum_{m n} d_{m n}\left(I_{x x}^{m n}+\partial_{x} I^{m n}\right)=\sum_{m>n}\left(d_{m n}+d_{n m}\right)(m-n)^{2} I^{m n} \\
& =\frac{f}{2 \pi}\left(-u_{k}^{2} \Sigma_{0}-\Sigma_{3}\right)
\end{aligned}
$$

where $\Sigma_{3}=2 \pi / f \sum_{m>n}\left(n_{n}^{0}-n_{m}^{0}\right) I^{m n} \simeq 1+\gamma x+\ldots$. In Eq. (7.7) we have taken only the first term of the expansion of $\Sigma_{3}$ in order to preserve the current conservation law. It was suggested in [20] that the diamagnetic part in Eq. (6.8) is not quite right. If taken correctly it will cancel the further expansion of $\Sigma_{\mathbf{3}}$. However, in any case, the term $D^{x x}$ affects neither the dispersion relation (6.13) nor the Meissner kernel (7.24).

The last term equals

$$
D_{y y}^{0}=\sum_{m n} d_{m n}\left(I_{y y}^{m n}+\frac{1}{2} x \partial_{x}^{2} I^{m n}\right)=\frac{f}{2 \pi} \Sigma_{2},
$$

which gives all elements of the matrix (7.7).

Similarly as presented above, the additional term for $u_{k}=0$ in Eq. (7.9) can be found.

\section{References}

[1] J.N. Leinass, J. Myrheim, Nuovo Cimento B 37, 1 (1977); A.B. Balachandran, Int. J. Mod. Phys. B 5, 2585 (1991).

[2] F. Wilczek, Phys. Rev. Lett. 49, 957 (1982); Int. J. Mod. Phys. B 5, 1273 (1991).

[3] D. Yoshioka, Prog. Theor. Phys. 81, 97 (1985); R. Prange, S. Girvin, The Quantum Hall Effect, Springer, Berlin 1990.

[4] A. Zee, Prog. Theor. Phys. Suppl. 107, 77 (1992).

[5] V. Kalmeyer, R.B. Laughlin, Phys. Rev. Lett. 59, 2095 (1987); R.B. Laughlin, Science 242, 525 (1988).

[6] B.I. Halperin, J. March-Russel, F. Wilczek, Phys. Rev. B 40, 8276 (1989).

[7] D. Arovas, J.R. Schrieffer, F. Wilczek, A. Zee, Nucl. Phys. B 251, 117 (1985). 
[8] E. Witten, Commun. Math. Phys. 117, 353 (1989); ibid. 121, 351 (1989);

D. Eliezer, G.W. Semenoff, Ann. Phys. 217, 66 (1992).

[9] X.G. Wen, F. Wilczek, A. Zee, Phys. Rev, B 39, 11413 (1989).

[10] A.V. Balatsky, Phys. Rev. B 43, 1257 (1991).

[11] J. March-Russel, F. Wilczek, Phys. Rev. Lett. 61, 2066 (1988).

[12] R.B. Laughlin, Phys. Rev. Lett. 50, 1395 (1983).

[13] B. Halperin, Phys. Rev. Lett. 52, 1583 (1984).

[14] J.K. Jain, Phys. Rev. Lett. 63, 199 (1989); ibid. Phys. Rev. B 40, 8079 (1990).

[15] M. Greiter, F. Wilczek, Mod. Phys. Lett. B 4, 1063 (1990).

[16] J.S. Birman, Ann. Math. Stud., Vol. 82, Braids, Links and Mapping Class Groups, Princeton Univ. Press, Princeton 1974.

[17] T. Einarsson, Phys. Rev. Lett. 64, 1995 (1990).

[18] F. Wilczek, Fractional Statistics and Anyon Superconductivity, World Scientific, Singapore 1990 .

[19] F. Wilczek, private communication.

[20] Y. Chen, F. Wilczek, E. Witten, B.I. Halperin, Int. J. Mod. Phys. B 3, 1001 (1989).

[21] A.L. Fetter, C.B. Hanna, R.B. Laughlin, Phys. Rev. B 39, 9679 (1989).

[22] P. Sitko, Phys. Lett. A 165, 363 (1992).

[23] A.L. Fetter, C.B. Hanna, R.B. Laughlin, Int. J. Mod. Phys. B 5, 2751 (1991).

[24] A.L. Fetter, private communication.

[25] S. Randjbar-Daemi, A. Salam, J. Strathdee, Nucl. Phys. B 340, 403 (1990).

[26] A.L. Fetter, C.B. Hanna, Phys. Rev. B 45, 2335 (1992).

[27] J.E. Hetrick, Y. Hosotani, B.-H. Lee, Ann: Phys. 209, 151 (1991).

[28] L. Bujkiewicz, L. Jacak, A. Radosz, to be published.

[29] F.D.M. Haldane, Phys. Rev. Lett. 67, 937 (1991). 